\title{
Carbon Footprint of Household Consumption of Different Income Groups_—Evidence from Micro-data of Chinese Households
}

\author{
Siyue Lai ${ }^{1}$, Xinyue Wang ${ }^{2}$, and Yuchen $\mathrm{Li}^{3}$ \\ ${ }^{1,2,3}$ Sichuan Agricultural University, Chengdu, China
}

\begin{abstract}
It is significant to discuss the carbon emissions in household consumption to achieve the goal of energy conservation and emission reduction. Based on the CLA analysis method, this paper calculates the carbon emissions of Chinese households in 2015 from a micro perspective, and analyzes the characteristics and structure of household carbon emissions at different income levels in different regions. The results show that most household carbon emissions come from direct energy, food, medical and daily necessities consumption, and the proportion of these three factors in household total carbon emissions gradually decreases with the increase of income level. At the regional level, the increase in income level does not necessarily lead to an increase in carbon emissions. Household carbon emissions and carbon emission structure fluctuate with income in a certain trend.
\end{abstract}

\section{Introduction}

Much greenhouse gas emissions are the primary cause of global climate change, and the key measures to slow down climate change are energy conservation and emission reduction. According to the World Energy Statistics Yearbook, China has become the largest carbon dioxide emitter in the world since 2006, of which $35 \%$ comes from household consumption ${ }^{[1]}$, and the carbon emissions generated by household consumption have become a new growth point of China's carbon emissions. Changing household consumption patterns is one of the effective ways to reduce emissions. The research shows that there are differences in carbon emissions between families in different regions and different income levels ${ }^{[2,3]}$. Paying attention to the characteristics and structure of carbon emissions caused by household consumption behaviors in different regions and different income levels can provide more targeted suggestions for the formulation of energy conservation and emission reduction policies.

$\mathrm{Qu}$ defines household $\mathrm{CO}_{2}$ emissions: "direct and indirect emissions of individuals or their families to meet the demands of their existence and development under certain socio-economic conditions ${ }^{[4]}$. Direct emissions refer to emissions related to household direct use of fuel, such as electricity, heating, natural gas, and other liquids. Indirect carbon emissions refer to the carbon emissions generated by indirect consumption of energy when households meet the needs of products and services. The family is the basic unit of society, and there are differences in lifestyle, scale, and income among families. This paper uses the consumer lifestyle approach (CLA) to calculate the related carbon dioxide emissions caused by various consumer behaviors.
The contribution of this paper has the following three points:

(1) Using the national survey data of China Household Finance Survey (CHFS) in 2015, this paper calculates the carbon emissions of household consumption in China from the micro perspective, and shows the carbon footprint and its distribution of Chinese households.

(2) Calculate and refine the carbon emission coefficients of 17 household consumption behaviors to estimate household carbon emissions more accurately.

(3) The sample data are divided into three regions and ten groups of income levels, analyzing the characteristics and structure of household consumption carbon emissions at different income levels in different regions

\section{Literature Review}

Household is the main unit of residents' living consumption, and the carbon emissions of household living consumption couldn't be underestimated. In the study of carbon emission estimation in the family dimension, most scholars analyze it from a macro perspective. Feng $\mathrm{Z} \mathrm{H}$ et al., Ji Zhiying and Duwei calculates the total carbon emissions of Chinese households $^{[5-7]}$. Macroeconomic data is difficult to accurately reflect the differences in carbon emissions between different countries or regions ${ }^{[8]}$. Wangyue puts forward that the research based on the micro-survey data of household consumption helps the authorities recognize the environmental benefits, which produced by different living consumption patterns ${ }^{[9]}$. Qu uses household survey data to study the carbon emission structure and its influencing factors of residents in Northwest China ${ }^{[4]}$, while Du Yunwei has studied the carbon emission

\footnotetext{
${ }^{*}$ Corresponding author: april332211@163.com
} 
characteristics and influencing factors of urban households in Jiangsu Province ${ }^{[10]}$.

Income level has a great influence on household carbon emissions. Duwei shows that the improvement of economic level is the main driving force of indirect carbon emissions of urban and rural residents ${ }^{[7]}$. In Ireland, direct household carbon emissions decrease with the increase of household income, while indirect emissions increase sharply with the increase of income ${ }^{[11]}$. In the United States and France, the richest $10 \%$ of the population emits three times as much direct carbon dioxide as the poorest $10 \%{ }^{[12]}$. Mi,Z calculated the carbon footprint of different income groups in China in 2012, and found that the top $5 \%$ of the population contributed $17 \%$ of the national household carbon footprint, while the lower $50 \%$ of the population contributed only $25 \%$, showing that there is a big gap in carbon emissions among different income groups ${ }^{[3]}$. In addition, income has different effects on household carbon emissions in different regions.

CLA was proposed by Dowlatabadi and Bin, which is used to measure the energy use and related $\mathrm{CO} 2$ emission activity expenses of American consumers ${ }^{[13]}$. From the consumption point of view, this method calculates the related carbon dioxide emissions caused by a certain household consumption behavior. Wei, Yang Ruihua, Xin Xukuo and Han Liyan have also calculated the carbon emissions of household consumption in China based on this method ${ }^{[2,14,15]}$. CLA considers the interaction factors affecting consumers, and combines the input-output method with the carbon emission coefficient method, which can better reflect the carbon emissions at the household level from a micro perspective.

This paper bases on the national survey data of CHFS in 2015, using the CLA method to analyze and calculate the carbon emissions, which generated by household consumption in China. And this paper analyzes the per capita carbon emissions of households with different income levels in three major regions of China.

\section{Data and methods}

\subsection{Methods}

Based on the CLA, the carbon emissions of residents' living consumption are calculated. According to the classification of household consumption by Wei and Wang Qinchi and the household energy consumption data used in this paper ${ }^{[14,16]}$, indirect household energy carbon emissions are divided into eight categories: food, clothing, housing, household equipment and services, medical and daily necessities, transportation and communication, education, culture and entertainment, and other goods and services. The specific calculation method is as follows:

First, estimate the carbon dioxide emissions from direct energy consumption:

$$
\mathrm{E}_{\text {direct }}=\sum f_{i j} * y_{i j}
$$

$\mathrm{E}_{\text {direct }}$ represents the total emissions from the $i$ th household's direct energy consumption, and $f_{j}$ is the direct carbon emissions intensity of the $j$ type direct energy consumption behavior, which is equal to the carbon dioxide emissions of each department divided by the total output of that department. $y_{i j}$ represents the expenditure of the $i$ th family on direct energy of $j$ type.

The carbon emissions generated by households in various types of consumption and services are obtained, and the equations (2) and (3) between the formulas are estimated

$$
\begin{aligned}
& \mathrm{E}_{\text {indirect }}=\sum T f_{i j} * y_{i j} \\
& T f=f *(\mathrm{E}-\mathrm{A})^{-1}
\end{aligned}
$$

$\mathrm{E}_{\text {indirect }}$ represents the total emissions from indirect consumption of the ith household, and $\mathrm{T} f_{j}$ is the carbon emissions intensity of class $j$ indirect consumption behavior. $y_{i j}$ represents the expenditure of the $i$ th family on direct energy of class $j$. $f$ is the vector of direct emission intensity in each production department, $\mathrm{A}$ is the matrix of domestic production coefficient, which is composed of direct consumption coefficient $a_{x y}$, and $a_{i j}$ represents the number of products in the $x$ department directly consumed by the production unit of the $y$ department, which is directly calculated from the inputoutput table. $(\mathrm{E}-\mathrm{A})^{-1}$ is Leontief inverse matrix, also known as the matrix of total demand coefficient, which indicates that one unit product produced by department $y$ consumes the complete demand of department $x$, including direct demand and indirect demand.

After calculating the carbon dioxide emission coefficients of various departments, the carbon emission coefficients of various consumption behaviors are obtained by corresponding the carbon emission coefficients of various departments. Finally, it is shown in Table 1.

Table 1. Classification of household energy consumption and

\begin{tabular}{|c|c|c|c|}
\hline Category & $\begin{array}{c}\text { Consumption } \\
\text { types }\end{array}$ & $\begin{array}{c}\text { Consumption } \\
\text { behavior }\end{array}$ & $\begin{array}{c}T f \\
\text { (ton/10000 } \\
\text { yuan) } \\
\end{array}$ \\
\hline \multirow{2}{*}{$\begin{array}{c}\text { Direct } \\
\text { consumption } \\
\text { of carbon } \\
\text { emissions }\end{array}$} & \multirow{2}{*}{ direct energy } & Heating & 6.504 \\
\hline & & fuel cost & 5.702 \\
\hline \multirow{10}{*}{$\begin{array}{l}\text { Indirect } \\
\text { consumption } \\
\text { of carbon } \\
\text { emissions }\end{array}$} & \multirow[b]{2}{*}{ food } & Board expenses & 0.706 \\
\hline & & $\begin{array}{l}\text { Consumption of } \\
\text { agricultural } \\
\text { products }\end{array}$ & 0.593 \\
\hline & clothing & Clothing & 0.837 \\
\hline & housing & $\begin{array}{l}\text { Decoration and } \\
\text { maintenance }\end{array}$ & 2.287 \\
\hline & \multirow{2}{*}{$\begin{array}{c}\text { household } \\
\text { equipment and } \\
\text { services }\end{array}$} & $\begin{array}{c}\text { household } \\
\text { electrical } \\
\text { appliances }\end{array}$ & 2.092 \\
\hline & & $\begin{array}{c}\text { Television fee } \\
\text { and network fee }\end{array}$ & 0.631 \\
\hline & \multirow{2}{*}{$\begin{array}{c}\text { medical and } \\
\text { daily } \\
\text { necessities } \\
\end{array}$} & Daily necessities & 2.014 \\
\hline & & Medical & 2.014 \\
\hline & & vehicle & 1.660 \\
\hline & & car fare & 1.799 \\
\hline
\end{tabular}
carbon dioxide emission coefficient of various consumption behaviors 


\begin{tabular}{|l|c|c|c|}
\hline & $\begin{array}{c}\text { transportation } \\
\text { and } \\
\text { communication }\end{array}$ & $\begin{array}{c}\text { Telephone } \\
\text { charges }\end{array}$ & 0.644 \\
\cline { 2 - 4 } & \multirow{2}{*}{$\begin{array}{c}\text { education } \\
\text { culture and } \\
\text { entertainment }\end{array}$} & $\begin{array}{c}\text { Cost of } \\
\text { education }\end{array}$ & 0.298 \\
\cline { 2 - 4 } & $\begin{array}{c}\text { Tourism } \\
\text { expenditure }\end{array}$ & 0.560 \\
\cline { 2 - 4 } & $\begin{array}{c}\text { other goods } \\
\text { and services }\end{array}$ & Domestic service & 0.698 \\
\cline { 3 - 4 } & Luxury & 1.806 \\
\hline
\end{tabular}

\subsection{Data sources and processing}

The data of household consumption expenditure in this paper comes from the data of China Household Finance Survey in 2015. The samples are distributed in 29 provinces (cities and districts, excluding Tibet, Xinjiang, and Taiwan). Based on the 2015 input-output table and China's carbon dioxide emissions in 2015 published by China Carbon Emissions Accounts Datasets (CEADs), the carbon dioxide emission coefficients of household consumption behaviors in 2015 are calculated. Finally, calculate the household carbon dioxide emissions.

In this paper, the outliers in the questionnaire data are truncated by $5 \%$. To make household consumption behaviors in China Carbon Emissions Accounts Datasets, input-output table and CHFS questionnaire correspond one by one, some industries in China Carbon Emissions Accounts Datasets are merged.

\section{Results and Analysis}

\subsection{The characteristics and structure of carbon emissions per capita of households with different income levels at the national level}

In this paper, the national sample is divided into ten groups according to the per capita income of families from small to large, and the per capita carbon emissions of families with different income levels are calculated. The per capita carbon emissions of households at the national level are 2.73 tons, of which indirect carbon emission and direct carbon emission are about 1.88 tons and 0.85 tons respectively. Indirect carbon emission is about 2.2 times of direct carbon emission, which is similar to the results obtained by Wei (2007).

With the increase of income level, the per capita carbon emissions of households increase step by step, and the growth rate is getting bigger and bigger. The per capita carbon emission of households with the lowest income $10 \%$ is about 1.58 tons, while that of households with the highest income $10 \%$ is about 5.41 tons, which is 3.43 times that of the former. The per capita carbon emissions of the families with the lowest income level increased slowly with the increase of income level, with an increase of about 0.15 tons. The carbon emissions of families in the highest income level increased greatly with income, which is 1.5 tons higher than that of the families with the highest second income level, with an increase of 1.58 tons. There is a big gap between the highest-income families and the lowest-income families in the total amount of carbon emissions per capita. Among all kinds of consumption carbon emission subcategories, the per capita carbon emissions of direct energy, food, transportation and communication increased by 0.97 tons, 0.73 tons and 0.63 tons respectively.

According to the carbon emission structure of residents of different income levels in China, the per capita carbon emission proportion of direct energy, food, medical and daily necessities consumption shows a downward trend. Among the households with the lowest income of $10 \%, 87.17 \%$ of the carbon emissions come from this three consumption. And among the households with the highest income of $10 \%$, these three accounts for $75.40 \%$ of the total carbon emissions. The proportion of carbon emissions from clothing, housing, transportation and communication, education, culture and entertainment, household equipment and services and other goods and services showed an upward trend, increasing from $12.81 \%$ to $24.6 \%$. However, the per capita carbon emission ratio of household consumption does not simply change linearly with the increase of income level. The per capita carbon emission ratio of nine types of consumption fluctuates with the increase of income level, and the increment of different types of consumption changes is also different.

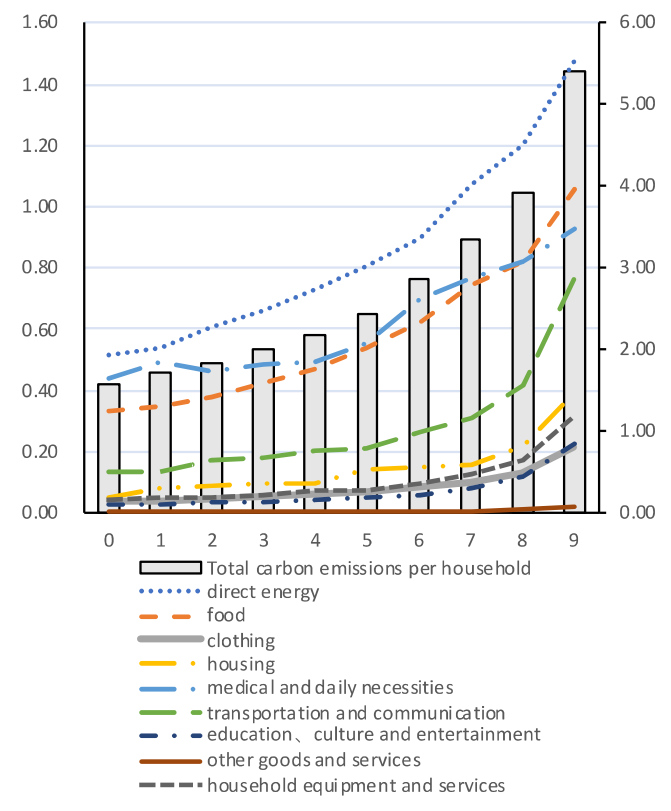

Fig. 1. Per capita carbon emissions of households across income levels 


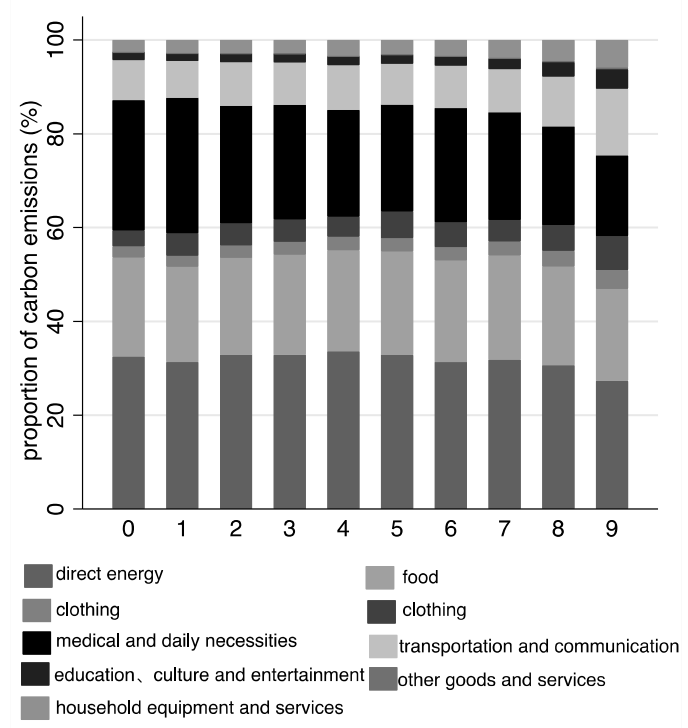

Fig. 2. The carbon emission structure of residents of various income levels across the country

This paper further divides the national sample into three regions: east, middle and west, and studies the characteristics and structure of carbon emissions per capita of families with different income levels in different regions.

\subsection{The characteristics and structural changes in household consumption carbon emissions at different income levels in different regions}

The results show that among families with the lowest income of $10 \%$, the per capita carbon emissions of the eastern, central, and western regions are 1.86 tons, 1.43 tons, and 1.27 tons respectively. The carbon emission of the top $10 \%$ of households in eastern China is 5.76 tons, while it is 4.85 tons in central China and 4.34 tons in western China. However, with the increase in income, the per capita carbon emission isn't necessarily increased. The per capita carbon emissions of families with an income of $10 \%-20 \%$ in the eastern region are lower than those with the lowest income of $10 \%$; The per capita carbon emissions of families with incomes of $30 \%-40 \%$ in western China are lower than those of their lower-level families. The carbon emissions of medical and daily necessities in eastern China are on the rise. The carbon emissions of direct energy, housing, medical care and daily necessities of households in the central region and six types of carbon emissions of direct energy, food, housing, medical care and daily necessities, transportation and communication, household equipment and services in the western region show a fluctuating upward trend with the increase of income level. The ratio of carbon emissions to total carbon emissions in the three regions also fluctuated with the increase of income.

The results of carbon emissions of households with different income levels in eastern, western and central China show that the increase of income level does not necessarily lead to the increase of carbon emissions, and the proportion of carbon emissions consumed by households in different regions does not necessarily increase or decrease with the increase of income.

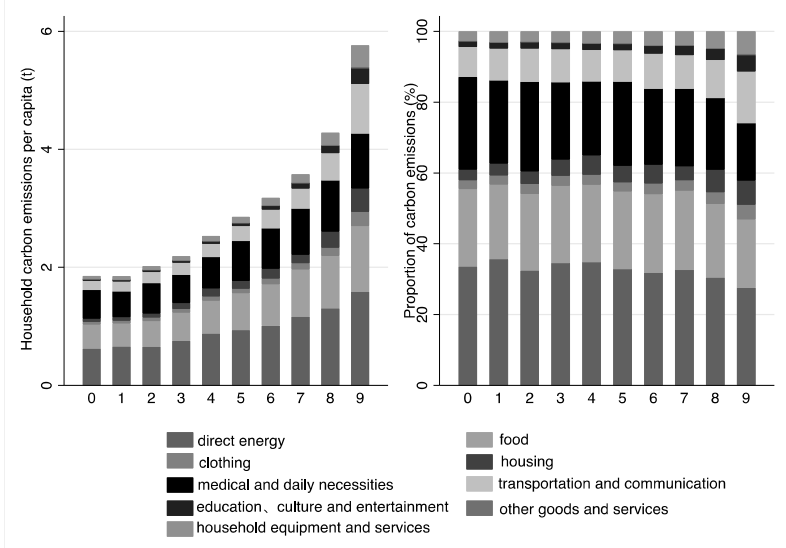

Fig. 3. Per capita carbon emissions of households in the eastern region

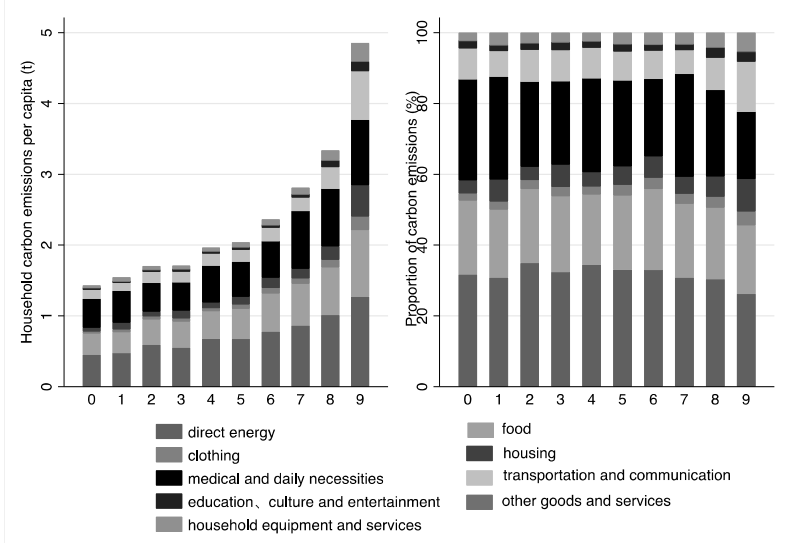

Fig. 4. Per capita carbon emissions of households in the central region

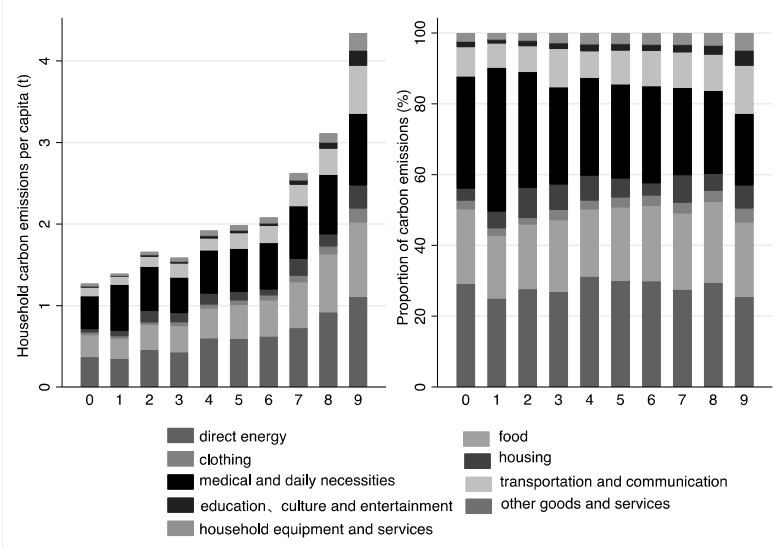

Fig. 5. Per capita carbon emissions of households in the western region

By analyzing the characteristics and structure of carbon emissions of households with different income levels in China and households with different income 
levels in different regions, it is found that the increase of income level does not necessarily lead to the increase of carbon emissions, and the changes of carbon emissions structure of households with different income levels are also inconsistent. Household carbon emissions and carbon emission structure do not change linearly with income, which indicates that the consumption patterns and behaviors of families in different income levels are also different, resulting in differences in consumption among families with different incomes.

\section{Conclusions}

This paper draws the following conclusions:

1. The per capita carbon emissions of households in China are 2.73 tons, of which indirect carbon emissions and direct carbon emissions are about 1.88 tons and 0.85 tons respectively. With the increase of income level, the per capita carbon emissions of households increase step by step, and the growth rate is getting more and more great.

2. Most household carbon emissions come from direct energy, food, medical and daily necessities. With the increase in income level, the proportion of the three factors in carbon emissions gradually decreased. For lowincome families, we should pay more attention to emission reduction measures of subsistence consumption, and for high-income families, we should advocate environmental protection and moderate lifestyle, and reduce enjoyment consumption.

3. At the regional level, the increase in income level does not necessarily lead to an increase in carbon emissions. Household carbon emissions and carbon emission structure do not change linearly with income but fluctuate under a certain trend. It shows that there are differences in consumption behavior among families with different income levels.

\section{References}

1. Tian X, Chang M, Lin C, et al. China's carbon footprint: A regional perspective on the effect of transitions in consumption and production patterns[J]. Applied Energy, 2014, 123:19-28.

2. Yang Ruihua, Ge Yousong, Zeng Hongying. Study on the characteristics of urban micro-household carbon emissions based on CLA model — Taking household carbon emissions questionnaire survey in 9 cities in China as an example [J]. Journal of Shanxi University: Natural Science Edition, 2011(04):149155.

3. Mi Z, Zheng J, Meng J, et al. Economic development and converging household carbon footprints in China $[\mathrm{J}]$. Nature Sustainability.

4. Qu J, Zeng J, Li Y, et al. Household carbon dioxide emissions from peasants and herdsmen in northwestern arid-alpine regions, China[J]. Energy Policy, 2013, 57:133-140.

5. FengZ H, Zou L L, Wei Y M. The impact of household consumption on energy use and $\mathrm{CO} 2$ emissions in China[J]. Energy, 2011, 36(1):656-670.
6. Ji Zhiying, Lai Xiaofeng, Jia Lijun. Carbon emissions from household energy consumption: measurement and driving factors $[\mathrm{J}]$. China Population Resources and Environment, 026(005):64-72.

7. Duwei. Analysis of indirect carbon emission characteristics of Chinese residents' lives based on LMDI decomposition model [J]. china population resources and environment, 2016(S2):14-18.

8. Qu Jiansheng, Wang Qin, Chen Fahu, et al. Assessment and Characteristic Analysis of Greenhouse Gas Emission in Gansu Province [J]. Development Research, 2008(3):55-58.

9. Wang Yue, Li Feng, Sun Xiao. Research progress of carbon emissions from urban household consumption [J]. Resources Science, 2019, 41(7): 1201-1212.

10. Du Yunwei, Huang Taozhen, Kang Guoding. Study on the characteristics and influencing factors of urban household carbon emissions from a micro perspective — Survey data from urban household activities in Jiangsu [J]. Population and Economy, 2015, 000(002):30-39.

11. Lyons S, Pentecost A, Tol R S J. Socioeconomic distribution of emissions and resource use in Ireland[J]. journal of environmental management, 2012, 112(none).

12. Chancel, L., 2014. Are younger generations higher carbon emitters than their elders: Inequalities, generations and $\mathrm{CO} 2$ emissions in France and in the USA. Ecol. Econ. 100, 195-207.

13. Bin S, Dowlatabadi H. Consumer lifestyle approach to US energy use and the related $\mathrm{CO} 2$ emissions[J]. Energy Policy, 2005, 33(2): p.197-208.

14. Wei YM, Liu LC, Fan Y, Wu G. The impact of lifestyle on energy use and $\mathrm{C} 02$ emission: an empirical analysis of China's households. Energy Policy 2007;35(1):247-57.

15. Xin Xu kuo, Han Liyan. micro evidence of how consumption patterns affect household carbon emissions from Chinese urban households [J]. southeast academic, 2017.

16. Wang Qinchi. Study on the Influence of Family Size on China's Energy Consumption and Carbon Emission [J]. Resources Science, 2015, 37(002):299307. 